\title{
Semantic visualisation in design computing
}

\author{
SIMEON J. SIMOFF AND MARY LOU MAHER \\ Key Centre of Design Computing and Cognition \\ University of Sydney, NSW 2006, Australia \\ simeon,mary@arch.usyd.edu.au
}

\begin{abstract}
In this paper we discuss the issues of visualisation in architectural design and civil engineering in the broader sense. Usually the term "visualisation" in these fields is connected with 3D modeling and photorealism of physical objects. Another part of the research community understands visualisation as the visual representation of numerical data, popular in the field of data mining and scientific visualisation. In this paper, we present a common approach for designing visualisation schemes that leverage pre-arranged semantics. The approach is based on a combination of the models of the metaphor in cognitive linguistics and the form-semantics-function categorisation. The use and development of metaphors is still handcrafted rather than based on formal methods. Our framework includes evaluation of the metaphor mapping.
\end{abstract}

\section{Introduction}

Visualisation, in broad sense, is the conversion of data into figures. This spans the area from simple 2D bar graphs and function curves to sophisticated graphical interaction in 3D virtual worlds and virtual reality systems. The latter are increasingly employed in design due to their emphasis on visualisation of geometry. From another point of view visualisation is a method of extracting meaningful information from complex objects using interactive graphics and imaging. In this case, through the means of visualisation techniques, data is converted into a consistent and, to some extent, compact graphical form, so that the observer can comprehend the knowledge and information in the data. Thus, visualisation, a process of mapping data onto visual dimensions to create a visual representation, is a key part of both data mining and virtual design paradigms.

In data mining, semantic visualisation can assist investigators to gain new insights from analysed data through the semantics of the visual form in which the data in presented. In computer-supported collaborative design environments, visualisation establishes the connection between the intended functionality of an object and the semantics of its form. For instance, the tools supporting conference activities in a conference room in the Virtual Campus at the University of Sydney have been assigned rectangular forms with a particular color code (Maher et al., 1999). In both cases, visualisation establishes the semantic connection between the form and the function associated with this form.

Design computing in architecture and civil engineering operates with an extensive amount of geometrical data. The geometric objects that are usually displayed and manipulated by CAD, 3D modelling and photorealism systems are the digital representations of the form of the objects that we model. For example, in architectural CAD, the 3D model of an arch looks like an arch. We also label it as an "arch" - a word that has particular meaning to us. The purpose, or the functionality of this arch can be different, depending on the context in which the model is used. If the model simulates part of physical reality - for example, a section of a bridge, then the function of the arch in that model is defined by the functionality of the modelled object from which the arch is part of. If the arch is an object within a virtual world, its functionality may have little in common with the functionality of physical arches in the engineering sense. For example, an arch in a virtual world can functionally be a gateway for taking an automated navigation path through a design project. 
We define semantic visualisation as a visualisation method, which establishes and preserves the semantic link between form and function in the context of the visualisation metaphor. Establishing a connection between form and functionality is not a trivial part of the design process. In a similar way, selecting the appropriate form for representing data graphically, whether the data consists of numbers or text, is not a straightforward procedure - the numbers or the text descriptions don't have a natural visual representation. On the other hand, how data are represented visually has a powerful effect on how the structure and hidden semantics in the data is perceived and understood.

We consider semantic visualisation for two derivatives of visualisation visibilisation and visistraction (Choras and Steinmann, 1995). Visibilisation is visualisation focusing on the presentation and interpretation which complies with rigorous mapping from physical reality. By contrast, visistraction is the visualisation of abstract concepts and phenomena, which do not have a direct physical interpretation or analogy. Visibilisation has the potential to bring key insights, by emphasising aspects that were unseen before. The dynamic visualisation of the heat transfer during the design of the heat-dissipating tiles cover of the underside of the space-shuttle is an early example of the application of visibilisation (Gore, 1981). Visistracton can give a graphic depiction of intuition regarding objects and relationships. The 4D simulation of data flow is an example of visistraction, which provides insights impossible without it. In a case-base reasoning system, visistraction techniques can be used to trace the change of relationships between different concepts with the addition of new cases.

Both kinds of visualisation play an important role in design computing. However, semantic visualisation remains a hand crafted methodology, where each case is considered separately. In this paper, we present an approach to semantic visualisation based on a cognitive model of metaphors, metaphor formalisation and evaluation. We illustrate the application of this approach with examples from visistraction of design communication data and visibilisation of virtual worlds.

\section{Form - Semantics - Function: a formal approach towards constructing and evaluating visualisation techniques for design computing.}

In semantic visualisation, we consider the form, semantics, and function of the objects used for visualisation of data. We present an approach to semantic visualisation based on:

- Metaphor analysis

- Metaphor formalisation

- Metaphor evaluation.

We draw our examples from visistraction, the visualisation techniques used to understand the results of data mining collaborative design sessions, and visibilisation, the visualisation of function in virtual worlds based on physical forms that have a similar function.

\section{METAPHOR ANALYSIS}

During metaphor analysis, the content of the metaphor is established. In the use of metaphor in cognitive linguistics, the terms source and target to refer to the conceptual spaces connected by the metaphor. The target is the conceptual space that is being described, and the source is the space that is being used to describe the target. In this mapping the structure of the source domain is projected onto the target domain in a way that is consistent with inherent target domain structure (Lakoff, 1993), (Turner, 1994). In

\footnotetext{
${ }^{\mathrm{i}}$ In the research literature the target is variously referred to as the primary system or the topic, and the source is often called the secondary system or the vehicle.
} 
the context of semantic visualisation, the consistent use of metaphor is expected to bring an understanding of a relatively abstract and unstructured domain in terms of more concrete and structured visual elements through the visualisation schemata.

An extension of the source-target mapping, proposed by (Turner and Fauconnier, 1995) includes the notion of generic space and blend space. Generic space contains the skeletal structure that applies to both source and target spaces. The blend space often includes structure not projected to it from either space, namely emergent structure on its own. The ideas and inspirations developed in the blend space can lead to modification of the initial input spaces and change the knowledge about those spaces, i.e. to change and evolve the metaphor. The process is called conceptual blending - it is the essence in the development of semantic visualisation techniques.

In our approach we combine the form-semantics-function categorisation of the objects being visualised with the Turner and Fauconnier (1995) model. The form of an object can express the semantics of that object, that is, the form can communicate implicit meaning understood through our experiences with that form. From the form in the source space we can connect to a function in the target space via the semantics of the form. The resultant model is shown in Figure 1, a particular case of the Turner and Fauconnier (1995) model applied to the visualisation of collaborative design data.

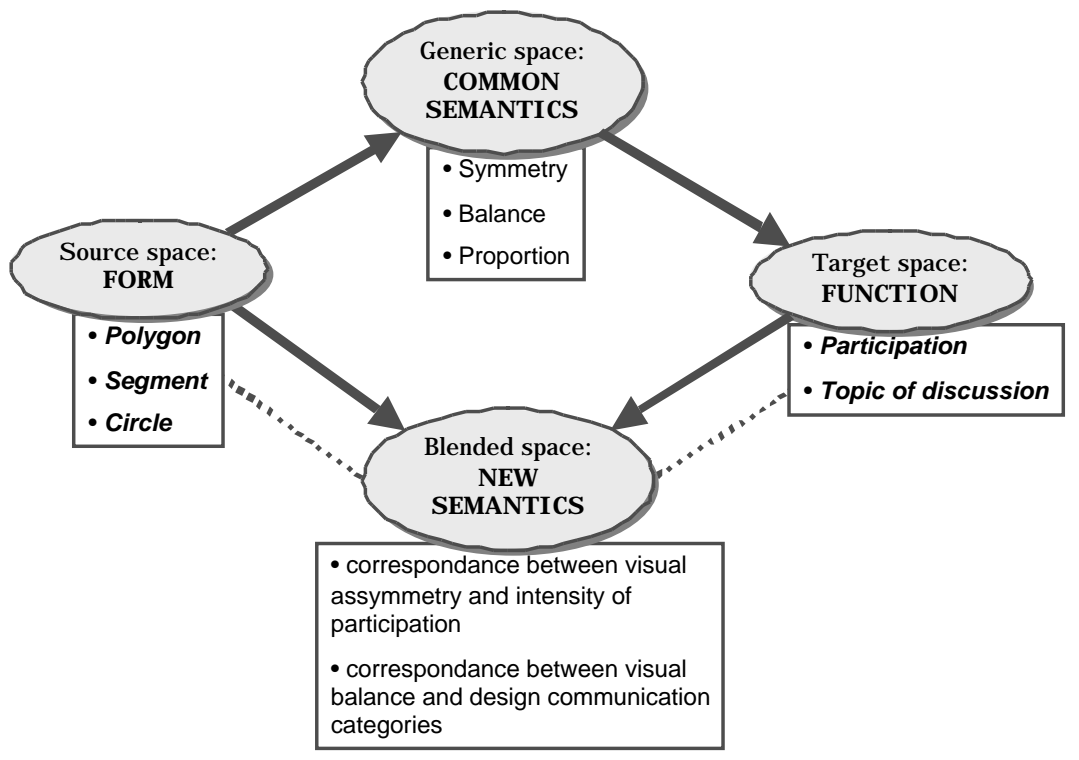

Figure 1. Metaphor analysis applied to the visualisation of collaborative design data.

The source space in this case is the space of 2D and 3D geometric shapes. The target space includes the concepts associated with the functions that we find in analysing a collaborative design session. The transfer of semantics has two components - the common semantics, which is carried by notions that are valid in both domains and what we call new semantics - the blend, which establishes the unique characteristics revealed by the correspondence between the form metaphor and functional characteristics of that form. The schema illustrates how metaphorical inferences produce parallel knowledge structures. The examples in the boxes under each space in Figure 1 are illustrated in Figure 2, which shows a visualisation of the results of data mining from a collaborative session in a textbased 3D virtual world. The discussion was one of many meetings of a design team in Active Worlds, a 3D collaborative modelling environment. The design project was the interior layout of a global learning building on the Stanford University campus. The data was categorised using a design communication coding scheme developed by Gabriel and Maher (1999). The visual metaphor of a polygon shape provides a mapping from the amount of utterances for each participant to a distance from a central point to the corresponding 
vertex of the polygon. The visual metaphor of a pie shape provides a mapping from the amount of discussion in each category to a proportion of the pie. The semantics of the polygon and the pie shape convey proportion and relative amounts. In Figure $2 \mathrm{a}$ the function each portion is mapped to in the target domain is the participant, when in Figure $2 \mathrm{~b}$ and $\mathrm{c}$ this function is the topic of the discussion. Without reading the specific numbers, $\mathrm{a}$ person can easily see that the project coordinator, together with designer's 1,3 and 5, were the most active participants. And, the majority of the discussion was in the category of design communication, with a high proportion of discussion about task/instruction, introduction of ideas, and clarification of ideas. Additionally, it is implicit in the pie visualisation that the sum of all categories of discussion is $100 \%$, that is, each statement was assigned only one category.

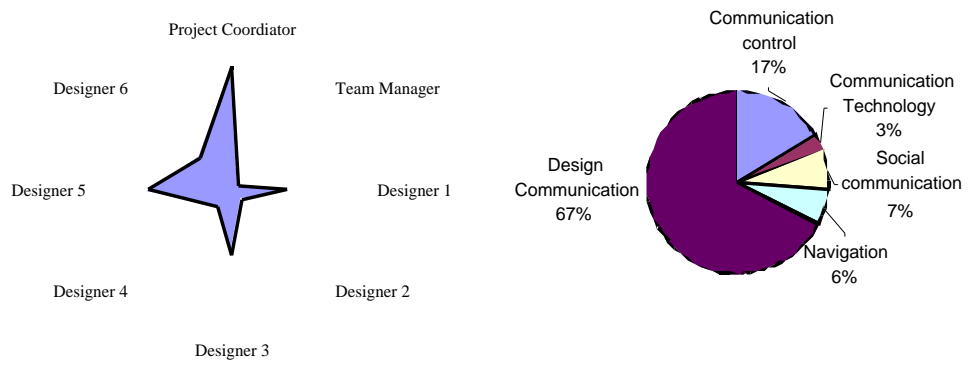

a. Individual participation b. Communication categories

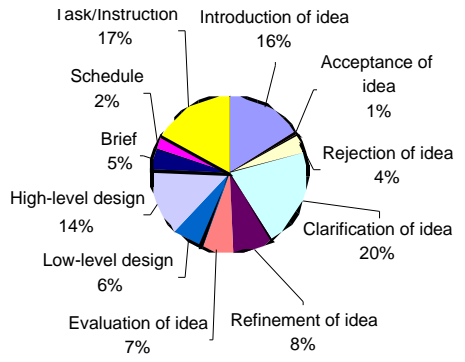

c. Design communication

Figure 2. Polygon shape visistraction of individual participation in team meeting and pie shape visistraction of categories and amounts of communication in team meeting.

\section{METAPHOR FORMALISATION}

Metaphor formalisation in the design of semantic visualisation schemes includes the following basic steps:

- Identification of the source and target spaces of the metaphor - the class of forms and the class of features or functions that these forms will represent. In the data mining of collaborative design discussions, this involves identifying the forms and the semantics that facilitate the visualisation of specific functions in the discussions. In the visualisation of virtual environments, this involves identifying the classes of forms of physical objects that will facilitate the understanding of functions of the virtual environments.

- Conceptual decomposition of the source and target spaces produces the set of concepts that describe both sides of the metaphor mapping. As a rule, metaphorical mappings do not occur isolated from one another. They are sometimes organized in hierarchical structures, in which 'lower' mappings in the hierarchy inherit the structures of the 'higher' mappings. In other words, this means that visualisation schemes, which use metaphor are expected to preserve the hierarchical structures of the data that they display. In visistraction, these are the geometric characteristics of the forms from the source space, and other form attributes like colours, line thickness, shading, etc. and the set of functions and features in the target space associated with these attributes and variations.

- Identifying the dimensions of the metaphor along which the metaphor operates. These dimensions constitute the common semantics. In visistraction this can be for instance key properties of the form, like symmetry and balance with respect to the center of gravity, that transfer semantics to the corresponding functional elements in the target domain. In the visualisation of virtual environments, these dimensions may act as 
constraints - for example, only the predefined elements with those geometrical properties can be used as the building blocks.

- Establishing semantic links, relations and transformations between the concepts in both spaces, creating a resemblance between the forms in the source domain and the functions in the target domain.

\section{METAPHOR EVALUATION}

In this section, we discuss the issues related to the evaluation of the visualisation. In spite of the large number of papers describing the use of the metaphor in the design of computer interfaces and virtual environments, there is a lack of formal evaluation. We have tailored the Anderson et al. (1994) model for investigating the metaphor mapping to meet our form-semantics-function formalism. The model is presented in Figure 3.

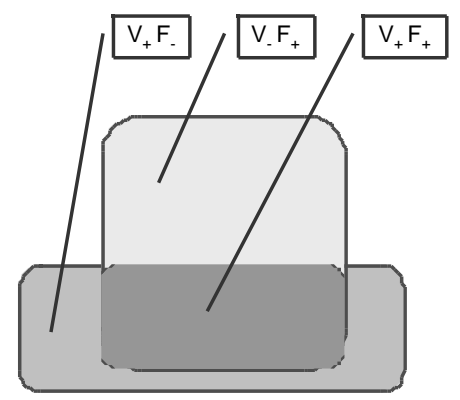

Figure 3. Model for evaluating metaphor mapping (based on (Anderson et al., 1994) )

The "V" and "F" are labels for visualisation and function features, respectively. The "VF" label with indices denotes numbers of features, namely:

- $V_{+} F_{+}$- function features that are mapped to the visualisation schema;

- $V_{-} F_{+}-$function features that are not supported by the visualisation schema;

- $V_{+} F_{-}$- features in the visualisation schema, not mapped to the functional features.

The ratio $\frac{V_{-} F_{+}}{V_{+} F_{+}}$provides an estimate of the quality of the metaphor used for the visualisation - the smaller the better. We illustrate the idea by evaluating examples of semantic visualisation of textual data and objects in virtual environments. In the first example, we consider two different forms for visualising the same set of functional features. In the second example we consider one form for conveying two different set of features.

The role of visistraction in concept relationship analyis is to assist the discovery of the relationship between concepts, as reflected in the available text data. The analysis uses word frequencies, their co-occurence and other statistics, and cluster analysis procedures. We investigate two visual metaphors - "Euclidian space" and "Tree", which provide a mapping from the numerical statistics and cluster analysis data into the target space (see Figure 1) of concepts and relations between them. The visualisation features for both metaphors and the function features of the target space are shown in Table 1. Examples of the two visualisation metaphors are shown in Figure 4. The example of Euclidian space metaphor, shown in Figure 4a, maps the statistics of words to coordinates of points in 2D/3D space. The blending semantics is the degree to which the terms are related to each other can be perceived visually from the distance between the corresponding data points the closer the points the tighter is the relationship between the words. However, it is 
difficult to make a steady judgement about what the relation is and whether we deal with complex terms. The distance to the surface, proportional to the frequency of the words can convey the message that a word is a key word. However, there is no feature in the visualisation, which shows context links between words, the strength of this links and other relations between words.

Table 1. Visualisation and function features

\begin{tabular}{|l|l|l|}
\hline $\begin{array}{l}\text { Visualisation features } \\
\text { of Euclidian space } \\
\text { metaphor }\end{array}$ & $\begin{array}{l}\text { Visualisation features of } \\
\text { tree metaphor }\end{array}$ & Function features \\
\hline - point & - nodes & - simple/complex concept \\
word point labels & - alphanumeric multi-word node & - subject key word \\
- axes & labels & - hierarchical relationship \\
- plane & - signs "+" and "-" & - link strength \\
- color & - branches & - synonymy \\
- line segment & - numeric labels for branches & - hyponymy \\
\hline
\end{tabular}
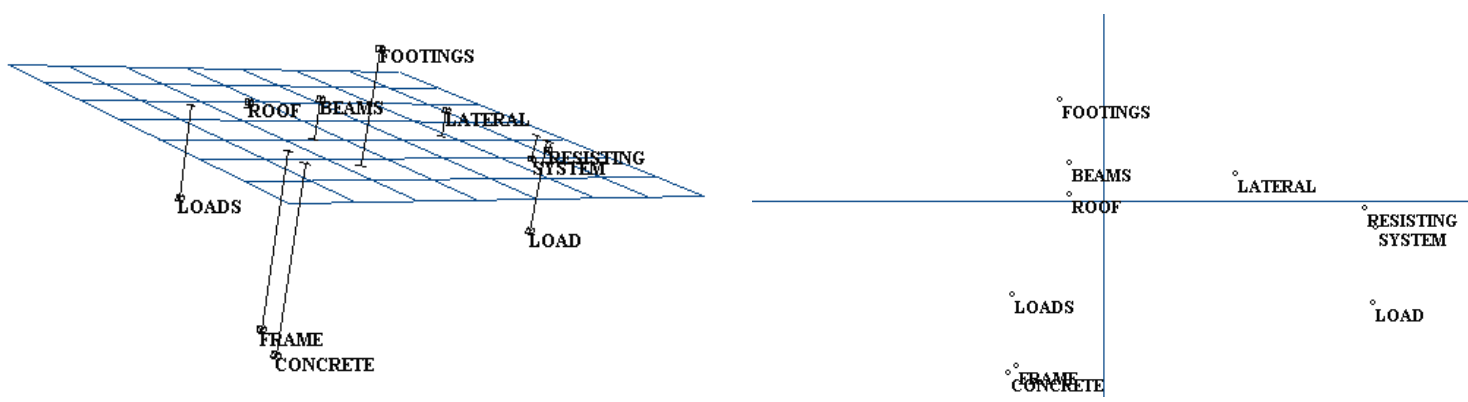

a. 10 most frequent words in the description of lateral load resisiting system in one of the wide-span building cases ${ }^{\text {ii }}$

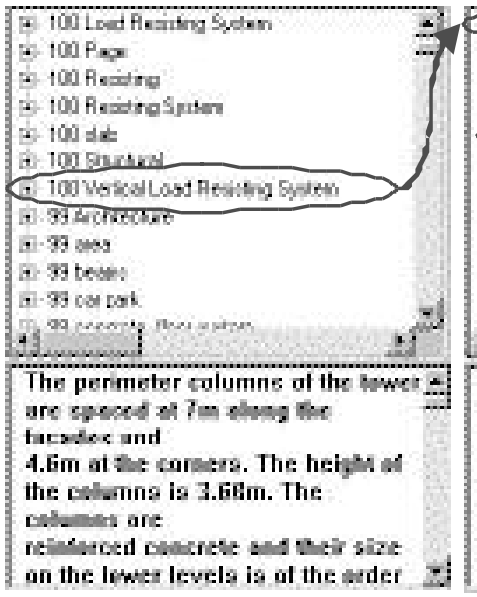

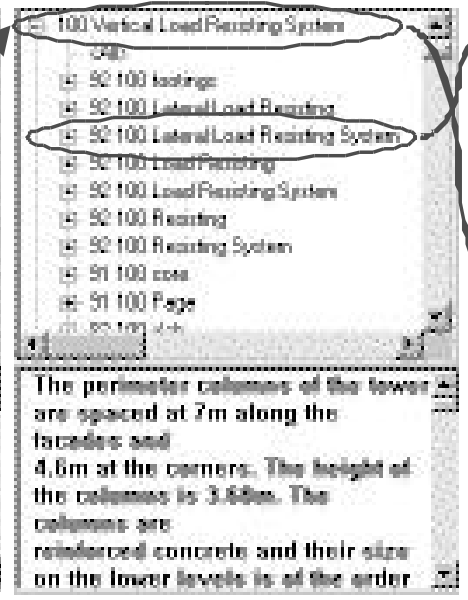

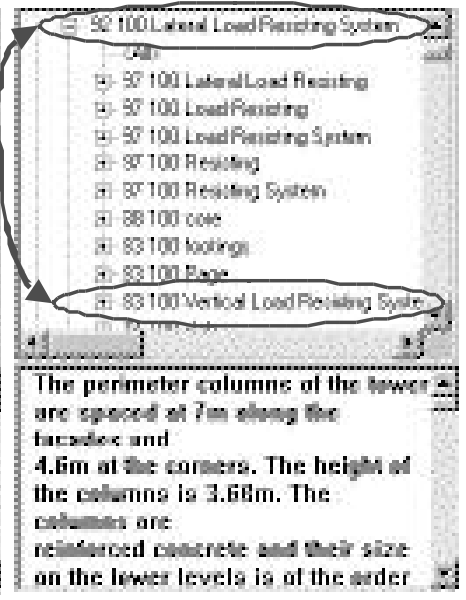

Part of a semantic net ${ }^{\mathrm{iii}}$ for the term "load resisting system".

Figure 4. Visistraction ualisation of the results of cluster analysis performed over a text data set ${ }^{\mathrm{iv}}$

\footnotetext{
${ }^{\text {ii }}$ CATPAC by Provalis Research Co.

iii TextAnalyst by Megaputer Intelligence, Inc.

${ }^{\text {iv }}$ The source text comes from the SAM (Structure and Materials) case library available at http://www.arch.usyd.edu.au/kcdc/caut/
} 
The example of a tree metaphor, shown in Figure $4 \mathrm{~b}$, maps the statistics of words to a topological structure of linked nodes. This visistaction includes nodes with single- and multiple-word labels, numeric values of each link between terms and the weight of the term among the other terms in the tree. The results of the comparison between the two metaphors are presented in Table 2 and Table 3. The Euclidean space metaphor has a poor performance for visistraction of concept relationships.

Table 2. Visualisation support for function features in Euclidean space and tree metaphors

\begin{tabular}{|l|c|c|}
\hline Function features & $\begin{array}{l}\text { Support by the } \\
\text { Euclidean space } \\
\text { metaphor }\end{array}$ & $\begin{array}{l}\text { Support by the Tree } \\
\text { metaphor }\end{array}$ \\
\hline Simple/complex concept & - & + \\
\hline Subject key word & + & + \\
\hline Hierarchical relationship & - & + \\
\hline Context link & - & + \\
\hline Link strength & - & - \\
\hline Synonymy & - & - \\
\hline Hyponymy & - & + \\
\hline
\end{tabular}

Table 3. Comparison of in Euclidean space and tree metaphors

\begin{tabular}{|c|c|c|}
\hline & $\begin{array}{l}\text { Euclidean } \\
\text { metaphor }\end{array}$ & Tree metaphor \\
\hline$V_{+} F_{+}$ & 1 & 5 \\
\hline$\overline{V_{-} F_{+}}$ & 6 & 2 \\
\hline$\frac{V_{-} F_{+}}{V_{+} F_{+}}$ & 6 & 0.4 \\
\hline
\end{tabular}

The other example of metaphor evaluation is connected with semantic visualisation of virtual environments. An example of visualisation and functional features under consideration is given in Table 4. In visualisation of virtual environments, we distinguish two cases:

- The virtual environment is used to visualise the elements of a design which is intended to be constructed physically, a visual simulation of a physical system. In this case we have a "thick" layer of common semantics in the face of the physical laws that establish the current limits of the functionality and a fairly thin blend space. The case presented in Figure 5a is a fragment of the virtual design of Stanford University Learning Lab. The arch is part of a building, which imples that it is intended to carry part of the load in the facade.

- The virtual environment is functional without being constructed physically, as in virtual worlds. The arches in Figure $5 \mathrm{~b}$ provide a visualisation of the entrance to information about architectural design styles in an Active Worlds universe. The arch form is used as a gateway towards particular part of the environment, providing perspective view and connection to the corresponding topic.

Table 4. Visualisation and function features

\begin{tabular}{|l|l|l|}
\hline $\begin{array}{l}\text { Visualisation } \\
\text { features }\end{array}$ & $\begin{array}{l}\text { Fuction features in virtual } \\
\text { environment for physical } \\
\text { simulation }\end{array}$ & $\begin{array}{l}\text { Function features in virtual } \\
\text { environment }\end{array}$ \\
\hline $\begin{array}{l}\text { - geometry } \\
\text { - size }\end{array}$ & $\begin{array}{l}\text { - carrying load in the building } \\
\text { - color } \\
\text { - texture }\end{array}$ & $\begin{array}{l}\text { - providing space under it } \\
\text { parts in the environment } \\
\text { - providing view to a } \\
\text { background information }\end{array}$ \\
\hline
\end{tabular}




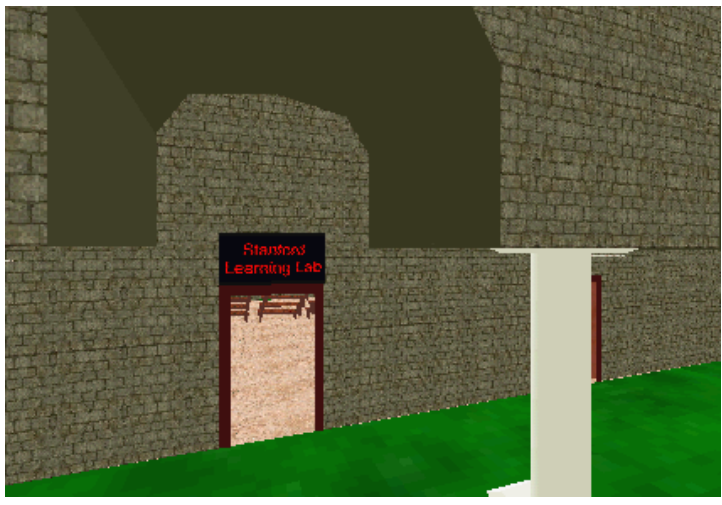

a.

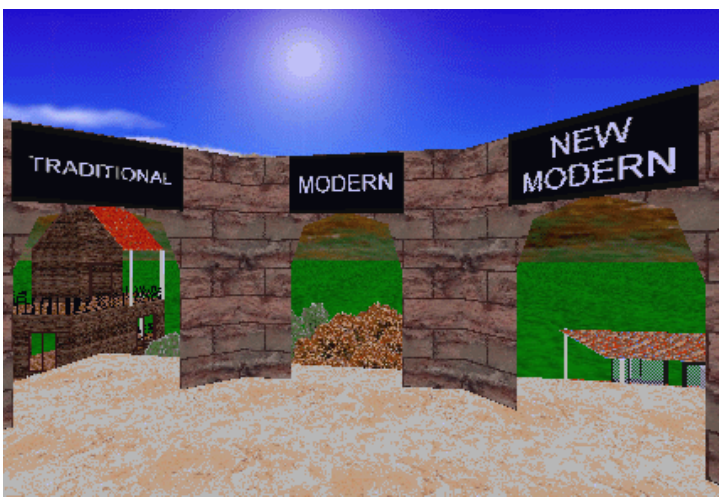

b.

Figure 5. Visualisation in a virtual design environment

\section{Conclusions}

The quality of the information presented to a designer during a design session is directly related to the facility in understanding the semantics of the data. Semantic visualisation can improve the quality of the presentation of large amounts of information. Visualisation, if used in an appropriate way, offers considerable semantic compression of presented information.

We present an approach for constructing semantic visualisation schemes, based on a metaphorical mapping in terms of form, semantics and functionality. We also illustrate the use of the approach for the selection of appropriate visualisation support in text data mining and semantic navigation in virtual design environments.

\section{Acknowledgements}

This research work is partially funded by the Australian Research Council

\section{References}

Anderson, B., Smyth, M., Knott, R. P., Bergan, M., Bergan, J. and Alty, J. L. (1994). Minimising conceptual baggage: Making choices about metaphor. In Cocton, G., Draper, S. and Weir, G. (eds), People and computers IX. Cambridge University Press, Cambridge, pp. 179-194.

Choras, D. N. and Steinmann, H. (1995). Virtual reality: Practical applications in business and industry, Prentice Hall, Upper Saddle River, New Hersey.

Gabriel, G. C. and Maher, M.L. (1999). Coding and modelling communication in architectural collaborative design, in O. Ataman and J Bermudez (eds) ACADIA '99, ACADIA, pp. 152-166.

Gore, R. (1981) In National Geographic, Vol. 159 , pp. 317-347.

Lakoff, G. (1993). The contemorary theory of metaphor. In Ortony, A. (ed.), Metaphor and thought, Cambridge University Press, Cambridge, pp. 202-251.

Maher, M. L., Simoff, S., Ning, G. and Lau, K. H. (1999) Two approaches to a virtual design office. In On-line Conference on Design Computing DCNet'99, http://www.arch.usyd.EDU.AU/kcdc/journal/.

Turner, M. (1994). Design for a theory of meaning. In Overton, W. and Palermo, D. (eds), The Nature and Ontogenesis of Meaning, Lawrence Erlbaum Associates, pp. 91-107.

Turner, M. and Fauconnier, G. (1995). Conceptual integration and formal expression, Journal of Metaphor and Symbolic Activity, 10, (3), 183-204.

${ }^{v}$ Active Worlds by Activeworlds.com, Inc. 